\title{
CONSTITUINTES QUÍMICOS DAS FOLHAS DE Qualea grandiflora: ATRIBUIÇÃO DOS DADOS DE RMN DE DOIS FLAVONÓIDES GLICOSILADOS ACILADOS DIASTEREOISOMÉRICOS
}

\author{
Mariane Cruz Costa Ayres, Samuel Portela Escórcio, Danielly Albuquerque da Costa e Mariana H. Chaves* \\ Departamento de Química, Universidade Federal do Piauí, 64049-550 Teresina - PI, Brasil \\ Gerardo Magela Vieira Júnior e Alberto J. Cavalheiro \\ Departamento de Química Orgânica, Instituto de Química, Universidade Estadual Paulista Júlio de Mesquita Filho, \\ 14800-900 Araraquara - SP, Brasil
}

Recebido em 30/10/07; aceito em 4/4/08; publicado na web em 4/9/08

\begin{abstract}
CHEMICAL CONSTITUENTS FROM LEAVES OF THE Qualea grandiflora: ATTRIBUTION OF THE NMR DATA OF TWO DIASTEREOISOMERIC ACYLATED FLAVONOID GLYCOSIDS. Phytochemical investigation from leaves of the Qualea grandiflora (Vochysiaceae) resulted in the isolation and identification of kaempferol-3- $O-\alpha-\mathrm{L}-(4$ "'-E-p-coumaroyl)-rhamnoside, kaempferol-3-O- $\alpha$-L-(4"'-Z-p-coumaroyl)-rhamnoside, squalene, phytol, lupeol, $\alpha$-amyrin, $\beta$-amyrin, sitosterol, sitostenone, sitosterol$3-O-\beta$-D-glucopyranoside, ursolic and oleanolic acids. The structures of the compounds were identified by $1 \mathrm{D}$ - and $2 \mathrm{D}-\mathrm{NMR}$ experiments, mass and UV spectrometry and comparison with literature data.
\end{abstract}

Keywords: Qualea grandiflora; Vochysiaceae; acylated kaempferol glycosids.

\section{INTRODUÇÃO}

Qualea grandiflora Mart. (Vochysiaceae) é uma planta de ocorrência em mata de galeria, cerrado e cerradão, considerada símbolo dos cerrados. ${ }^{1}$ É conhecida popularmente como pau-terra, pau-terra-do-campo, pau-terra-do-cerrado, pau-terra-da-folha-larga, ariavá, entre outros. ${ }^{2}$ As cascas e folhas são medicinais e os frutos dão matéria tintorial amarela. ${ }^{2}$ Infusão ou decocção das folhas de $Q$. grandiflora são usadas no tratamento de diarréia com sangue, cólicas intestinais e contra ameba. ${ }^{3}$ As cascas são usadas, na forma de infusão, para limpeza externa de úlceras e feridas e no tratamento de doenças inflamatórias. ${ }^{1}$ Segundo levantamento etnobotânico (dados não publicados), realizado no município de Nazaré do Piauí-PI, a infusão preparada com frutos desta espécie é usada no tratamento da asma.

O extrato etanólico das cascas de Q. grandiflora exibe ação antiulcerogênica ${ }^{4}$ e o metanólico tem ação antibacteriana sobre o Staphylococcus aureus, Bacillus cereus e Pseudomonas aeruginosa. ${ }^{5}$ O extrato etanólico das folhas tem efeito antioxidante, ${ }^{6}$ analgésico, depressor do sistema nervoso central, com potencial anticonvulsivante ${ }^{7}$ e ação antibacteriana sobre o Staphylococcus epidermidis (ATCC 12228 e MRSE H-111). ${ }^{8}$

O presente artigo relata pela primeira vez o estudo químico do extrato etanólico das folhas de $Q$. grandiflora, do qual foram isolados e identificados o kanferol-3-O- $\alpha$-L-(4"- $E$ - $p$-cumaroil)-raminosideo (1), kanferol-3-O- $\alpha$-L-(4"-Z-p-cumaroil)-raminosideo (2), esqualeno, fitol, lupeol, $\alpha$-amirina, $\beta$-amirina, sitosterol, sitostenona, ácidos

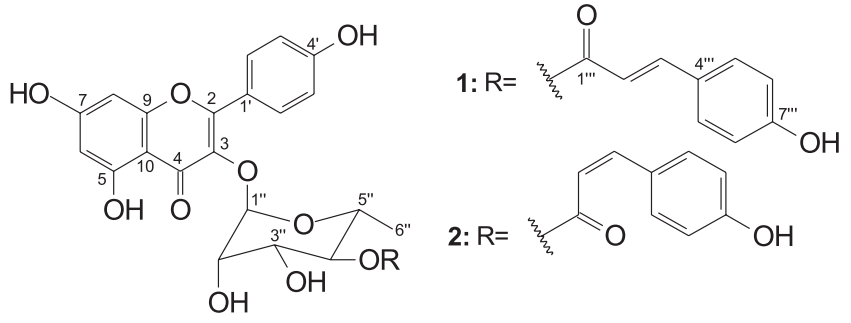

Figura 1. Estrutura dos flavonóides isolados das folhas de Q. grandiflora

\footnotetext{
*e-mail: mariana@ufpi.br
}

ursólico e oleanólico e 3-O- $\beta$-D-glicopiranosídeo do sitosterol.

\section{PARTE EXPERIMENTAL}

\section{Procedimentos experimentais gerais}

Os espectros de RMN ${ }^{1} \mathrm{He} \mathrm{e}^{13} \mathrm{C}$ foram obtidos em espectrômetro Brüker modelo Avance DRX-500 e DPX 300 e Varian Inova 500 operando a 500 e $300 \mathrm{MHz}\left({ }^{1} \mathrm{H}\right), 125$ e $75 \mathrm{MHz}\left({ }^{13} \mathrm{C}\right)$, utilizando $\mathrm{CD}_{3} \mathrm{OD}$ ou $\mathrm{CDCl}_{3}$ como solventes e TMS como referência interna. As placas cromatográficas foram preparadas utilizando uma mistura de gel de sílica 60 G Vetec e $60 \mathrm{GF}_{254}$ Fluka (1:1) e as revelações das cromatoplacas foram feitas por nebulização com solução de $\mathrm{Ce}\left(\mathrm{SO}_{4}\right)_{2}$ e com vapores de iodo. Os solventes, etanol, metanol, hexano, acetato de etila, clorofórmio e diclorometano, utilizados na preparação dos extratos e fracionamentos cromatográficos clássicos, foram todos de grau analítico obtidos da Vetec. Para cromatografia líquida de alta eficiência em escala preparativa (CLAE-Prep), foi utilizado o seguinte equipamento: cromatógrafo preparativo Varian, modelo Prep star SD-1, com detector UV-VIS modelo 320, injetor manual Rheodyne modelo 7725i com alça de amostra de $2,5 \mathrm{~mL}$, aquisição de dados via computador tipo Pentium III $550 \mathrm{MHz}$ utilizando software Varian Star Chromatography Workstation, versão 5.31. Coluna Phenomenex - Luna fenil hexil, (250 x 21,20 mm, $10 \mu \mathrm{m}$ ), sem pré-coluna. Os espectros de massas foram obtidos em espectrômetro VG Platform da Fisons Instruments.

\section{Material vegetal}

Folhas de Q. grandiflora foram coletadas no Município de Demerval Lobão-Piauí, em abril de 1998 e identificadas no Herbário Graziela Barroso - UFPI, onde se encontra depositada a exsicata, com o número TEPB 10.091.

\section{Isolamento dos constituintes químicos}

As folhas foram secas ao ar, moídas (1000 g) e extraídas exaustivamente com etanol a $95 \%$. O volume do extrato etanólico, equi- 
valente a $98 \mathrm{~g}$, determinado por meio do peso seco, foi reduzido a $300 \mathrm{~mL}$, por concentração em evaporador rotativo, adicionado 600 $\mathrm{mL}$ de água e submetido à partição com acetato de etila, obtendo-se as frações aquosa (23,2 g), AcOEt e uma interfase (7,7 g). A fase AcOEt foi concentrada, suspensa em $\mathrm{MeOH}-\mathrm{H}_{2} \mathrm{O}$ (9:1) e submetida à partição com hexano, resultando nas fases hidroalcoólica $(48,1 \mathrm{~g})$ e hexânica $(9,5 \mathrm{~g})$. A fase hidroalcoólica $(10 \mathrm{~g})$ foi fracionada em coluna filtrante $(25 \times 5,0 \mathrm{~cm})$ preenchida com $140 \mathrm{~g}$ de gel de sílica, eluída com $\mathrm{CHCl}_{3}-\mathrm{MeOH}$ (5-100\%), resultando em 40 frações de $150 \mathrm{~mL}$, coletadas como segue: frações 1-6 $\left(\mathrm{CHCl}_{3}-\mathrm{MeOH}, 95: 5\right)$, 7-17 ( $\left.\mathrm{CHCl}_{3}-\mathrm{MeOH}, 9: 1\right), 18-28\left(\mathrm{CHCl}_{3}-\mathrm{MeOH}, 8: 2\right), 29-35\left(\mathrm{CHCl}_{3}-\right.$ $\mathrm{MeOH}, 1: 1)$ e 36-40 (MeOH 100\%). A fração H-10 (227 mg) foi recromatografada em coluna de Sephadex LH-20 eluída com MeOH fornecendo uma mistura de flavonóides $(56 \mathrm{mg}$ ) os quais foram separados em CLAE-UV DAD, eluída no modo gradiente exploratório $\mathrm{H}_{2} \mathrm{O}+\mathrm{HAc}-0,5 \% / \mathrm{ACN}$ (5-100\%); 200-600 nm; $1 \mathrm{~mL} / \mathrm{min} ; 40 \mathrm{~min}$. Após condicionamento para o modo isocrático, com múltiplas injeções, usando como eluente $\mathrm{H}_{2} \mathrm{O} / \mathrm{ACN}$ (60:40), resultou na separação de $\mathbf{1}(24 \mathrm{mg})$ e $\mathbf{2}(8 \mathrm{mg})$.

Kanferol-3-O- $\alpha$-L-(4"-E-p-cumaroil)-raminosideo (1): UV $\lambda_{\mathrm{m}}$ $(\mathrm{MeOH}) \mathrm{nm}: 215,266$ e 311. EM-IES: $m / z$ (int. rel) $578\left[\mathrm{M}^{++}\right], 285$ (100), 286. RMN ${ }^{1} \mathrm{H} \mathrm{e}^{13} \mathrm{C}$ ( $\left.\mathrm{CD}_{3} \mathrm{OD}\right)$ : Tabela 1.

Kanferol-3-O- $\alpha-L-\left(4\right.$ "-Z-p-cumaroil)-raminosideo (2): UV $\lambda_{\text {m }}$ $(\mathrm{MeOH}) \mathrm{nm}: 265$ e 316 EM-IES: $\mathrm{m} / z$ (int. rel) $578\left[\mathrm{M}^{\circ}\right]$, 285, 286 (100). RMN ${ }^{1} \mathrm{H} \mathrm{e}^{13} \mathrm{C}\left(\mathrm{CD}_{3} \mathrm{OD}\right)$ : Tabela 1.

A fase hexânica da partição, depois de concentrada, foi suspensa em $\mathrm{MeOH}$ a quente e refrigerada originando um precipitado graxo $(7,2 \mathrm{~g})$ e o material solúvel em $\mathrm{MeOH}(1,7 \mathrm{~g})$. Este último foi fracionado em coluna de gel de sílica (36 x 2,6 cm), eluída com hexano-AcOEt (0-100\%) e AcOEt-MeOH (1:1) resultando em 68 frações (S-1 a S-68), coletadas como segue: frações 1-4 (hexano 100\%), 5-12 hexano-AcOEt, 98:2), 13-15 (hexano-AcOEt, 95:5), 16-26 (hexano-AcOEt, 9:1), 27-40 (hexano-AcOEt, 8:2), 41-43 (hexano-AcOEt, 7:3), 44-52 (hexano-AcOEt, 6:4), 53-57 (hexanoAcOEt, 1:1), 58-59 (AcOEt 100\%) e 60-68 AcOEt-MeOH, 1:1). Após evaporação do solvente em evaporador rotativo e análise por CCD, as frações coletadas foram reunidas em 11 grupos. O grupo $\mathrm{S}-2$ (fração $2,14 \mathrm{mg}$ ) correspondeu ao esqualeno. Os grupos S-16 (frações 16-19, $169 \mathrm{mg}$ ) e S-20 (frações 20-24, $262 \mathrm{mg}$ ) foram recromatografados, o primeiro em Sephadex LH-20 com hexano- $\mathrm{CH}_{2} \mathrm{Cl}_{2}$ (1:4) fornecendo $48 \mathrm{mg}$ de uma mistura de fitol, lupeol, $\alpha$ - e $\beta$-amirina, o segundo grupo foi em coluna de gel de sílica, eluída de modo isocrático com $\mathrm{CHCl}_{3}-\mathrm{MeOH}$ (98:2), resultando na sitostenona (6 mg) e na fração $\mathrm{S} 21-7$, que foi recristalizada em $\mathrm{MeOH}$ fornecendo o sitosterol (100 $\mathrm{mg}$ ). O grupo S-35 (frações 35-36, $40 \mathrm{mg}$ ) foi recromatografado em coluna de Sephadex LH-20, eluída com $\mathrm{CH}_{2} \mathrm{Cl}_{2}$-acetona (3:2), resultando em $9 \mathrm{mg}$ de uma mistura dos ácidos ursólico e oleanólico. $\mathrm{O}$ tratamento desta mistura com excesso de $\mathrm{CH}_{2} \mathrm{~N}_{2}$, em banho de gelo, conduziu aos respectivos derivados metilados $(10 \mathrm{mg})$. O grupo $\mathrm{S}-60$ (frações 60-62, $603 \mathrm{mg}$ ) foi recromatografado em coluna de gel de sílica eluída com $\mathrm{CHCl}_{3}-\mathrm{MeOH}(0-10 \%)$ originando F16-30 (61 mg). $\mathrm{O}$ tratamento desta fração com anidrido acético $(1 \mathrm{~mL})$ em presença de piridina $(1 \mathrm{~mL})$ à temperatura ambiente por $12 \mathrm{~h}$, forneceu, após filtração em gel de sílica, o tetracetado de 3-O- $\beta$-D-glicopiranosídeo do sitosterol $(30 \mathrm{mg})$.

\section{RESULTADOS E DISCUSSÃO}

O esqualeno, ${ }^{9}$ fitol, ${ }^{10}$ lupeol,,${ }^{11} \alpha$-amirina, ${ }^{11} \beta$-amirina, ${ }^{11}$ sitosterol, ${ }^{12}$ sitostenona, ${ }^{13}$ ácidos ursólico e oleanólico ${ }^{14}$ e 3-O- $\beta$-Dglicopiranosídeo do sitosterol ${ }^{15}$ foram isolados do material solúvel em metanol, resultante da fase hexânica da partição do extrato etanólico das folhas de $Q$. grandiflora. Estas substâncias são comuns em outras espécies, portanto suas estruturas foram identificadas por comparação dos dados espectroscópicos de RMN ${ }^{1} \mathrm{H}$ e ${ }^{13} \mathrm{C}$ obtidos com os relatados na literatura. ${ }^{9-15}$

$\mathrm{O}$ fitol, lupeol, $\alpha$ - e $\beta$-amirina foram isolados em mistura e identificados por meio da análise dos espectros de $\mathrm{RMN}{ }^{1} \mathrm{H} \mathrm{e}{ }^{13} \mathrm{C}$. O fitol apresentou-se como o constituinte majoritário, sendo evidenciado no espectro de $\mathrm{RMN}{ }^{1} \mathrm{H}$ pelos sinais em $\delta 4,16$ (d), atribuído aos hidrogênios oximetilênicos e alílicos, e $\delta 5,42(\mathrm{t})$, referente ao hidrogênio olefínico. Esta análise foi confirmada pela observação no espectro de $\mathrm{RMN}{ }^{13} \mathrm{C}$ dos sinais em $\delta 59,7\left(\mathrm{CH}_{2} \mathrm{O}\right), 123,4$ e 140,5 (CH e C olefínicos). ${ }^{10} \mathrm{O}$ espectro de $\mathrm{RMN}{ }^{1} \mathrm{H}$ da mistura apresentou ainda um duplo dupleto característico de triterpenóides $3 \beta-\mathrm{OH}(\delta 3,23$; $J=11,0$ e $5,2 \mathrm{~Hz} ; \mathrm{H}-3)$, sendo confirmado no espectro de RMN ${ }^{13} \mathrm{C}$, pela presença do sinal $\delta 79,5$ (C-3). ${ }^{11,16}$ A ocorrência do lupeol, $\alpha$ - e $\beta$-amirina foi determinada de acordo com a metodologia descrita na literatura para análise de mistura de triterpenóides, devido à existência de três pares de sinais em $\delta 109,6 / 151,2 ; 125,5 / 138,0 ; 122,0 / 145,5$ característicos dos carbonos olefínicos nos esqueletos triterpênicos lupano, ursano e oleanano, respectivamente. ${ }^{16}$

O 3-O- $\beta$-D-glicopiranosídeo do sitosterol foi identificado como derivado tetracetilado. ${ }^{15}$ Os ácidos ursólico e oleanólico, após serem metilados com diazometano, foram identificados em mistura, na razão de 67:33. Esta proporção foi determinada no espectro de RMN ${ }^{1} \mathrm{H}$ pela comparação das integrações dos sinais dos hidrogênios olefínicos em $\delta 5,18$ e 5,21, atribuídos ao H-12 no ursolato e oleanolato de metila, respectivamente, com a do sinal em $\delta 3,62(J=10,8$ e 4,7 $\mathrm{Hz}$ ), referente ao H-3 dos dois triterpenóides. ${ }^{17}$ Este espectro apresentou ainda, entre outros sinais, um simpleto em $\delta 3,62$ referente aos hidrogênios metoxílicos, confirmando a ocorrência da reação de metilação. $\mathrm{O}$ espectro de $\mathrm{RMN}{ }^{13} \mathrm{C}$ apresentou sinais de carbonos metoxílico $(\delta 51,5)$ e carbonílico $(\delta 178,1)$ de éster, de carbono oximetínico $(\delta 79,0, \mathrm{C}-3)$, além de dois pares de sinais em $\delta 125,6 / 138,1$ e 122,3/144,0 atribuídos aos carbonos olefínicos C-12 e C-13 nos esqueletos triterpênicos ursano e oleanano, respectivamente. ${ }^{16}$ Todos os dados de RMN observados foram consistentes com os relatados para o ursolato e oleanolato de metila. ${ }^{14}$

Os flavonóides $\mathbf{1}$ e $\mathbf{2}$, isolados da fase hidroalcoólica, foram identificados utilizando os dados dos espectros de massas, RMN 1D e $2 \mathrm{D} \operatorname{COSY}{ }^{1} \mathrm{H}-{ }^{1} \mathrm{H}, \mathrm{HMQC}$ e $\mathrm{HMBC}$.

$\mathrm{O}$ espectro de $\mathrm{RMN}{ }^{1} \mathrm{H}$ de $\mathbf{1}$ apresentou, na região de hidrogênios aromáticos, um par de dupletos em $\delta 6,21$ e $6,37(\mathrm{~J}=2,0 \mathrm{~Hz})$, característico de sistema $\mathrm{AB}$, correspondente a dois hidrogênios metaposicionados e atribuídos, respectivamente, a H-6 e H-8 de flavonóis. Os dois pares de dupletos em $\delta$ 7,01 e 7,76 $(\mathrm{J}=8,7 \mathrm{~Hz}) ; \delta$ 7,52 e 6,83 $(\mathrm{J}=8,6 \mathrm{~Hz})$, indicativos de sistemas do tipo AA'BB', caracterizaram dois anéis aromáticos para-dissubstituídos. ${ }^{18}$ Além destes, o espectro apresentou um par de dupletos em $\delta 6,29$ e 7,56 $(J=16 \mathrm{~Hz})$ de um sistema de hidrogênios olefínicos com configuração $E$ e sinais de hidrogênios ligados a carbonos oximetínicos $(\delta 3,21-5,60)$ de uma unidade osídica. ${ }^{18}$ O dupleto em $\delta 0,79\left(\mathrm{~J}=6,2 \mathrm{~Hz}, \mathrm{CH}_{3}\right)$ juntamente com o sinal em $\delta 5,50$ $(J=1,6 \mathrm{~Hz}, \mathrm{CH})$, referente ao hidrogênio anomérico, sugeriu a existência de uma unidade de $\alpha$-raminose.$^{18} \mathrm{O}$ mapa de contorno $\operatorname{COSY}{ }^{1} \mathrm{H}-{ }^{1} \mathrm{H}$ confirmou todos os acoplamentos observados no espectro de RMN ${ }^{1} \mathrm{H}$ unidimensional e permitiu evidenciar as correlações dos sinais dos hidrogênios em $\delta$ 5,50 (H-1") com $\delta 4,23$ (H-2"'), do sinal de H-2", $\operatorname{com} \delta 3,92$ (H-3"), de H-3" com $\delta 4,94$ (H-4"), de H-4" com $\delta 3,21$ (H-5") e de H-5" com $\delta$ 0,79 (H-6"). Esta análise juntamente com os dados de RMN ${ }^{13} \mathrm{C}$ e mapas de contornos bidimensionais HMQC e HMBC sugeriu tratar-se de um raminopiranosideo do kanferol com uma das hidroxilas da raminose esterificando o ácido $p$-cumárico. $\mathrm{O}$ EM de 1 apresentou o pico do íon molecular a $\mathrm{m} / \mathrm{z} 578$, consistente com a fórmula molecular $\mathrm{C}_{30} \mathrm{H}_{26} \mathrm{O}_{12}$, e o pico base a $\mathrm{m} / z 285$ indicativo da unidade de kanferol. ${ }^{19} \mathrm{~A}$ localização da raminose no C-3 do 
Tabela 1. Dados de RMN ${ }^{1} \mathrm{H}(500 \mathrm{MHz})$ e ${ }^{13} \mathrm{C}(125 \mathrm{MHz})$ de $\mathbf{1}$ e 2, incluindo resultados de mapas de contornos de correlação heteronuclear ${ }^{1} \mathrm{H}-{ }^{13} \mathrm{C}-\mathrm{COSY}{ }^{\mathrm{n}} J_{\mathrm{CH}}(\mathrm{n}=1, \mathrm{HMQC} ; \mathrm{n}=2$ e $3, \mathrm{HMBC})$, em $\mathrm{CD}_{3} \mathrm{OD}$. Deslocamentos químicos em $\delta(\mathrm{ppm})$ e constantes de acoplamento $(J$, entre parêntese) em $\mathrm{Hz}$

\begin{tabular}{|c|c|c|c|c|c|c|c|c|}
\hline \multirow[b]{3}{*}{$\mathrm{C}$} & \multicolumn{4}{|c|}{1} & \multicolumn{4}{|c|}{2} \\
\hline & \multicolumn{2}{|c|}{ HMQC } & \multicolumn{2}{|c|}{ HMBC } & \multicolumn{2}{|c|}{ HMQC } & \multicolumn{2}{|c|}{ HMBC } \\
\hline & $\delta_{\mathrm{C}}$ & $\delta_{H}$ & ${ }^{2} J_{\mathrm{CH}}$ & ${ }^{3} J_{\mathrm{CH}}$ & $\delta_{\mathrm{C}}$ & $\delta_{H}$ & ${ }^{2} J_{\mathrm{CH}}$ & ${ }^{3} J_{C H}$ \\
\hline 2 & 159,6 & & & H-2'/H-6' & 159,4 & & & H-2'/H-6' \\
\hline 3 & 135,4 & & & $\mathrm{H}-1$, & 135,7 & & & $\mathrm{H}-1 "$ \\
\hline 4 & 178,6 & & & & 179,5 & & & \\
\hline 5 & 163,4 & & H-6 & & 163,2 & & H-6 & \\
\hline 6 & 100,2 & $6,21(\mathrm{~d}, 2,0)$ & & H-8 & 100,1 & $6,21(\mathrm{~d}, 2,0)$ & & H-8 \\
\hline 7 & 166,5 & & H-8, H-6 & & 166,3 & & H-8, H-6 & \\
\hline 8 & 95,1 & $6,37(\mathrm{~d}, 2,0)$ & & H-6 & 94,9 & $6,40(\mathrm{~d}, 2,0)$ & & H-6 \\
\hline 9 & 158,8 & & H-8 & & 158,7 & & $\mathrm{H}-8$ & \\
\hline 10 & 106,1 & & & H-6, H-8 & 105,9 & & & H-6, H-8 \\
\hline $1^{\prime}$ & 122,8 & & & H-3'/H5' & 122,6 & & & H-3'/H-5' \\
\hline $2^{\prime} 6^{\prime}$ & 132,1 & $7,76(\mathrm{~d}, 8,7)$ & & & 131,9 & $7,73(\mathrm{~d}, 8,5)$ & & \\
\hline 3,5 & 116,7 & $7,01(\mathrm{~d}, 8,7)$ & & & 116,6 & $6,94(\mathrm{~d}, 8,5)$ & & \\
\hline $4^{\prime}$ & 161,9 & & H-3'/H-5' & H-2'/H-6' & 161,7 & & H-3'/H-5' & H-2'/H6' \\
\hline $1 "$ & 102,3 & $5,50(\mathrm{~d}, 1,6)$ & & & 102,7 & $5,50(\mathrm{~d}, 1,5)$ & & \\
\hline $2 "$ & 71,9 & $\begin{array}{c}4,23(\mathrm{dd}, 1,6 \\
\text { e } 3,2)\end{array}$ & & & 71,9 & $\begin{array}{c}4,23(\mathrm{dd}, 2,0 \\
\text { e } 3,5)\end{array}$ & & \\
\hline $3 "$ & 70,3 & $\begin{array}{c}3,92(\mathrm{dd}, 3,2 \\
\text { e } 9,7)\end{array}$ & $\mathrm{H}-4$ " & $\mathrm{H}-1$ ", & 70,2 & $\begin{array}{c}3,89(\mathrm{dd}, 3,0 \\
\text { e } 9,5)\end{array}$ & $\mathrm{H}-4 "$ & \\
\hline $4 "$ & 74,9 & $4,94(t, 9,7)$ & H-3", H-5" & H-2", H-6" & 74,5 & $4,90(t, 9,5)$ & H-3", H-5" & H-2", H-6" \\
\hline $5 "$ & 69,8 & $3,21(\mathrm{~m})$ & H-4", H-6" & & 69,8 & $3,30(\mathrm{~m})$ & H-4", H-6" & $\mathrm{H}-1 "$ \\
\hline $6 "$ & 17,8 & $0,79(\mathrm{~d}, 6,2)$ & & $\mathrm{H}-4$ " & 17,6 & $0,79(\mathrm{~d}, 6,5)$ & & $\mathrm{H}-4 "$ \\
\hline 1 '” & 168,9 & & $\mathrm{H}-2$ "” & H-3"', H-4" & 167,7 & & & H-3"', H-4" \\
\hline $2 "$, & 115,3 & $6,29(\mathrm{~d}, 16,0)$ & H-3", & & 116,4 & $5,75(\mathrm{~d}, 13,0)$ & H-3", & \\
\hline $3 "$, & 146,9 & $7,56(\mathrm{~d}, 16,0)$ & & H-5"'/H-9"' & 145,6 & $6,87(\mathrm{~d}, 13,0)$ & & H-5"'/H-9", \\
\hline $4 "$, & 127,4 & & & $\begin{array}{c}\text { H-2"', H-6- } \\
\text { "'/H-8"," }\end{array}$ & 127,6 & & & 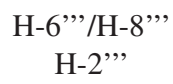 \\
\hline $5, " 9, "$ & 131,5 & $7,52(\mathrm{~d}, 8,6)$ & & $\mathrm{H}-3^{\prime \prime}$ & 133,9 & $7,66(\mathrm{~d}, 8,5)$ & & $\mathrm{H}-3^{\prime \prime}$ \\
\hline $6 ", 8 "$, & 116,9 & $6,83(\mathrm{~d}, 8,6)$ & & & 115,8 & $6,74(\mathrm{~d}, 8,5)$ & & \\
\hline 7"” & 161,4 & & H6"”/H-8"" & H-5" "/H-9", & 160,1 & & H6"”/H-8", & H-5"'/H-9", \\
\hline
\end{tabular}

kanferol foi evidenciada pela desblindagem de 9,6 ppm para C-3 em relação ao da aglicona,$^{20}$ sendo confirmada pela correlação do sinal em $\delta$ 135,4 (C-3) com $\delta 5,50(d, 1,6 \mathrm{~Hz})$, referente ao hidrogênio anomérico (H-1"). Definiu-se a localização da unidade $p$-cumaroíla, por meio da observação no mapa de contorno HMBC, da correlação $\left({ }^{3} J\right)$ entre o sinal em $\delta 4,94$, atribuído a H-4" da raminose, e $\delta 168,9$ referente à carbonila (C-1"') da unidade $p$-cumaroíla. Todos os dados espectroscópicos observados e inequivocamente atribuídos (Tabela 1) são consistentes com a estrutura do kanferol-3-O- $\alpha-\mathrm{L}-(4$ "'-E-pcumaroil)-raminosideo (1).

Os espectros de RMN e de massas da substância 2 mostraram-se semelhantes aos de $\mathbf{1}$, porém a principal diferença observada foi no espectro de RMN ${ }^{1} \mathrm{H}$, pois os hidrogênios etilênicos, H-2"'e H-3"', encontram-se mais blindados, ficando situados em $\delta 5,75$ e 6,87, respectivamente. A configuração $Z$ foi deduzida pelo menor valor da constante de acoplamento $(13 \mathrm{~Hz})$ dos hidrogênios etilênicos em relação à observada para a configuração $E(16 \mathrm{~Hz})$, mostrando-se concordantes com o relatado para outros flavonóides glicosilados acilados. ${ }^{18}$ Todos os dados obtidos (Tabela 1) são consistentes com a estrutura do kanferol-3-O- $\alpha$-L-(4"-Z-p-cumaroil)-raminosideo (2).

O flavonóide 1 foi relatado na espécie Ocotea vellosiana, ${ }^{21}$ pertencente à família Lauraceae e a mistura de $\mathbf{1 + 2}$ na Philotheca pinoides (Rutaceae), ${ }^{22}$ contudo os dados de RMN só foram publicados parcialmente, portanto este artigo descreve pela primeira vez as atribuições inequívocas dos deslocamentos químicos de RMN ${ }^{1} \mathrm{H}$ e ${ }^{13} \mathrm{C}$ para os flavonóides diastereoisoméricos $\mathbf{1}$ e 2.

$\mathrm{O}$ isolamento de flavonóides a partir do extrato etanólico das folhas de $Q$. grandiflora pode justificar em parte a atividade antioxidante deste extrato, relatada anteriormente. ${ }^{6}$

O estudo realizado com a $Q$. grandiflora contribui para o conhecimento da fitoquímica da família Vochysiaceae e está de acordo com o relatado para outras plantas desta família, as quais apresentam flavonóides, triterpenóides e esteróides, além de taninos, benzoquinonas e antraquinonas.

\section{AGRADECIMENTOS}

Ao CNPq, CAPES e FINEP pela bolsa (M. H. Chaves e D. A. Costa) e apoio financeiro, à Dra. G. M. Sousa do Herbário Graziela Barroso, UFPI, pela identificação do material botânico, ao Centro Nordestino de Aplicação e Uso da Ressonância Magnética Nuclear (CENAUREMN-UFC) 


\section{REFERÊNCIAS}

1. Almeida, S. P.; Proença, C. E. B.; Sano, S. M.; Ribeiro, J. F.; Cerrado: espécies vegetais úteis, EMBRAPA-CPAC: Distrito Federal, 1998.

2. Corrêa, M. P.; Dicionário de Plantas Úteis do Brasil e exóticas cultivadas, Ministério da Agricultura/Instituto Brasileiro de Desenvolvimento Florestal: Rio de Janeiro, 1978, vol. 5.

3. Rodrigues, V. E. G.; Carvalho, D. A.; Ciências Agrotecnológicas 2001, $25,102$.

4. Hiruma-Lima, C. A.; Santos, L. C.; Kushima, H.; Pellizzon, C. H.; Silveira, G. G.; Vasconcelos, P. C. P.; Vilegas, W.; Souza Brito, A. R. M.; J. Ethnopharmacol. 2006, 104, 207.

5. Alves, T. M. A.; Silva, A. F.; Brandão, M.; Grandi, T. S. M.; Smânia, E. F. A.; Smânia Jr., A.; Zani, C. L.; Mem. Inst. Oswaldo Cruz 2000, 95, 367.

6. Sousa, C. M. M.; Silva, H. R.; Vieira-Júnior, G. M.; Ayres, M. C. C.; Costa, C. L. S.; Araújo, D. S.; Cavalcante, L. C. D.; Barros, E. D. S.; Araújo, P. B. M.; Brandão, M. S.; Chaves, M. H.; Quim. Nova 2007, 30, 351.

7. Gaspi, F. O. G.; Foglio, M. A.; Carvalho, J. E.; Moreno, R. A.; J. Ethnopharmacol. 2006, 107, 19.

8. Ayres, M. C. C.; Brandão, M. S.; Vieira Júnior, G. M.; Menor, J. C. A. S.; Silva, H. B.; Soares, M. J. S.; Chaves, M. H.; Rev. Bras. Farmacogn. 2008, 18, 90.

9. Breitmaier, E.; Voelter, W.; Carbon-13 NMR spectroscopy: high resolution methods and applications in Organic Chemistry and Biochemistry, $3^{\text {rd }}$ ed., VCH: New York, 1987.
10. Rahman, A.; Ahmad, V. U.; ${ }^{13}$ C-NMR of Natural Products: Diterpenes, Plenum Press: New York, 1992.

11. Mahato, S. B.; Nandy, A. K.; Roy, G.; Phytochemistry 1992, 31, 2199.

12. De-Eknamkul, W.; Potduang, B.; Phytochemistry 2003, 62, 389.

13. Chaves, M. H.; Roque, N. F.; Ayres, M. C. C.; J. Braz. Chem. Soc. 2004, 15, 608; Tandon, S.; Rastogi, R. P.; Planta Med. 1976, 29, 190.

14. Piozzi, F.; Paternostro, M.; Passannanti, S.; Gacs-Baitz, E.; Phytochemistry 1986, 25, 539.

15. Fang, J.- M., Wang, K.- C.; Cheng, Y.- S.; Phytochemistry 1991, 30, 3383

16. Olea, R. S. G.; Roque, N. F.; Quim. Nova 1990, 13, 278.

17. Vieira Júnior, G. M.; Souza, C. M. L.; Chaves, M. H.; Quim. Nova 2005, 28, 183; Vieira Júnior, G. M.; Carvalho, A. A.; Gonzaga, W. A.; Chaves, M. H.; Quim. Nova 2007, 30, 491.

18. Fiorini, C.; David, B.; Fourasté, I.; Vercauteren, J.; Phytochemistry 1998, 47, 881.

19. Pizzolatti, M. G.; Cunha Jr., A.; Szpoganicz, B.; Sousa, E.; Braz-Filho, R.; Schripsema, J.; Quim. Nova 2003, 26, 466.

20. Agrawal, P. K., ed; Carbon-13 NMR of flavonoids, Elsevier: Amsterdam, 1989.

21. Garcez, W. S.; Yoshida, M.; Gottlieb, O. R.; Phytochemistry 1995, 39, 815.

22. Sultana, N.; Sarker, S. D.; Armstrong, J. A.; Wilson, P. G.; Waterman, P. G.; Biochem. Syst. Ecol. 2003, 31, 681. 